\title{
Full counting statistics of Cooper pair shuttling
}

\author{
Alessandro Romito \\ NEST-INFM \& Scuola Normale Superiore, Piazza dei Cavalieri 7, I-56126 Pisa, Italy \\ Yu. V. Nazarov \\ Department of Nanoscience, Delft University of Technology, 2628 CJ Delft, The Netherlands
}

(Received 16 February 2004; published 28 December 2004)

\begin{abstract}
The Cooper pair shuttle is a simple model system that combines features of coherent and incoherent transport. We evaluate the full counting statistics (FCS) of charge transfer via the shuttle in the incoherent regime. We describe two limiting cases when the FCS allows for classical interpretation. Generally, the classical interpretation fails, yielding negative and imaginary "probabilities." This signals that superconducting coherence survives even in the incoherent regime. We evaluate the current noise in some detail.
\end{abstract}

DOI: 10.1103/PhysRevB.70.212509

PACS number(s): 74.50.+r, 74.40.+k, 03.65.Yz

The Josephson effect ${ }^{1}$ consists in coherent Cooper pair transfer through a tunnel junction between two bulk superconductors. It results in a dissipationless current present as a ground state property of the system. This is in contrast to dissipative electron transfer in normal conductors. In the latter case, it is possible to evaluate the full counting statistics (FCS) of charge transfers ${ }^{2}$ in terms of classical probabilities. Thereby, the transport properties are fully understood and characterized: one can predict not only the average current, but also the current noise and all higher moments of the current distribution function. ${ }^{3}$

One can also access the FCS of a superconducting Josephson junction. ${ }^{4}$ Due to gauge symmetry breaking in the superconducting state, this FCS cannot be interpreted in classical terms yielding negative "probabilities." The use and the interpretation of the FCS in this case is that it determines the quantum evolution of the detector that measures the current. ${ }^{5}$

A recent proposal puts forward an interesting way to transfer Cooper pairs: to shuttle them (controllably) between the superconducting electrodes. ${ }^{6}$ Although the original proposal puts emphasis on mechanical degrees of freedom, a Cooper pair shuttle is essentially a superconducting single electron transistor (SSET) with variable time-dependent Josephson coupling to the superconducting leads. ${ }^{7}$ The Coulomb island of this SSET is brought in contact with one electrode at a time, the electrode contacted being periodically altered. $^{8}$

It is interesting that the supercurrent between the leads is achieved as a result of a nonequilibrium driven process, generally accompanied by dissipation. The coherence of Cooper pairs transferred is determined by the coherence of different charge states in the island, this being mostly affected by fluctuations of the gate voltage. ${ }^{7}$ The transport results from the interplay between the coherent Josephson coupling and decoherence effects. This is why the Cooper pair shuttle presents a model to bridge between the limits of coherent and incoherent transport. The model is simple indeed; one can restrict the consideration to just two quantum states.

Thus motivated, we have analyzed the FCS of the Cooper pair shuttle in the most interesting incoherent regime, where no net supercurrent is shuttled. The regime is achieved in the limit when the voltage fluctuations are classical. If the fluc- tuations come from an environment, this implies that the temperature of the environment exceeds the relevant energy scales of the shuttle. Naively, from the absence of the net current one would conclude that no charge transfer occurs in the system. However, transfers do occur, the current is zero only in average, and the FCS presents a convenient way to reveal this circumstance. This stipulates the understanding of transport properties of the shuttle and, generally, the interplay between coherent and incoherent transport.

The results are as follows. If the period of the shuttling is sufficiently long for decoherence to be accomplished, the FCS can be interpreted in terms of classical elementary events: Cooper pair transfers. During the shuttling cycle, either no transfer takes place or a pair is transferred in either direction. There is an apparent similarity with the FCS of the pumping in normal systems studied in Refs. 9 and 10. The FCS in the opposite limit of short cycles allows for alternative classical interpretation in terms of a superconducting current randomly switching between two opposite values.

In the general intermediate situation, the FCS cannot be interpreted in classical terms. An attempt to evaluate the probabilities per cycle yields negative and even imaginary values. This is a clear signature of the fact that the superconducting coherence survives strong dephasing although this coherence does not manifest itself in a net superconducting current. It was recently explained ${ }^{11}$ that any FCS can be characterized directly so one would not have to measure higher cumulants of the current noise one by one. However, the immediate physical value measured would also depend on the properties of the concrete detector. This is also a way to experimentally observe the FCS of the Cooper pair shuttle.

The system of interest is presented schematically in Fig. 1. It consists of a Cooper pair box, or Coulomb island, connected with Josephson junctions to the superconducting leads $L$ and $R$. Conventionally, ${ }^{6-8}$ we assume the separation of energy scales, $\Delta \gg E_{C} \gg E_{J}, \Delta$ being the superconducting energy gap, $E_{C}$ being the charging energy of the box, and $E_{J}$ being a typical Josephson junction energy. Under these conditions the quasiparticle degrees of freedom are not involved in the system's dynamics. In addition, the gate voltage is chosen to bring two charge states of the box, say, $|0\rangle$ and $|1\rangle$, 


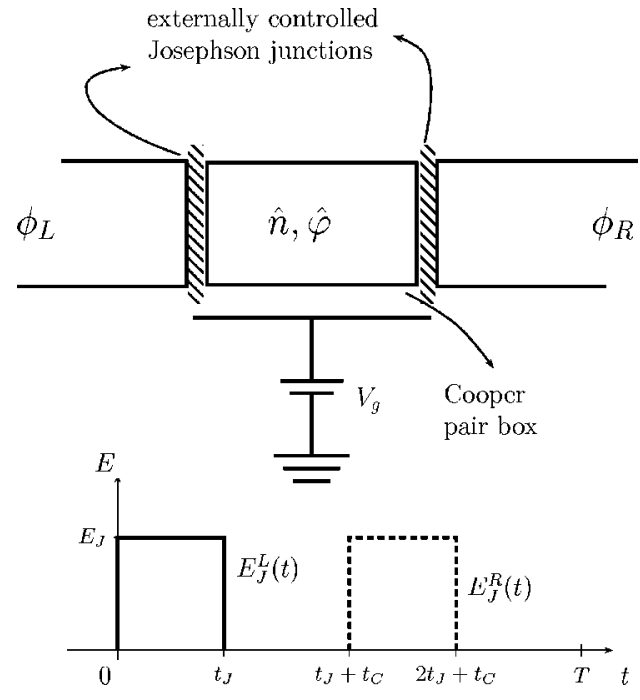

FIG. 1. Upper panel: Cooper pair shuttle consists of a Cooper pair box coupled to superconducting leads through Josephson junctions. Two charge states are tuned to the degeneracy point by the gate voltage. Lower panel: The specific time dependence of $E_{J}^{(R, L)}$ within a single period $T$ provides the shuttling.

to the degeneracy point. Under these assumptions, we can restrict the consideration to these two states only and the shuttle is described by the Hamiltonian

$$
\hat{H}(t)=2 e V(t) \sigma_{z}-\sum_{b=L, R} \frac{E_{J}^{(b)}(t)}{2}\left(e^{i \phi_{b}} \sigma_{+}+\sigma_{-} e^{-i \phi_{b}}\right),
$$

which we write in terms of $2 \times 2$ Pauli matrices $\sigma_{z}, \sigma_{ \pm}$ $\equiv\left(\sigma_{x} \pm \sigma_{y}\right) / 2$ in the space spanned by $|0\rangle,|1\rangle . V(t)$ is proportional to the deviation of the gate voltage from the value corresponding to the degeneracy point and $\phi_{R}, \phi_{L}$ are the phases of the macroscopic superconductors. Only $\phi=\phi_{R}$ $-\phi_{L}$ is a physical quantity which we will assume to be fixed; this can be obtained by closing the circuit end points in a loop pierced by a constant magnetic flux. $E_{J}^{(L, R)}(t)$ are timedependent Josephson energies of the left and right junctions. For the sake of concreteness, we assume stepwise periodic variation of both Josephson energies with time as shown in the lower panel of Fig. 1. Each lead is contacted during time interval $t_{J}$ and the box contacts no lead during time interval $t_{C}$ for each period $T=2\left(t_{J}+t_{C}\right)$. We call $t_{J}$ and $t_{C}$ the "Josephson contact" and the "free evolution" time, respectively. An idea to realize the required time dependence in Josephson coupling by means of an experimentally realizable setup based on superconducting quantum interference devices has been proposed in Ref. 7 .

We assume $V(t)$ to be a classical stochastic variable with white noise statistics $\langle V(t)\rangle_{\text {stoc }}=V_{0}$ and $\left\langle V(t) V\left(t^{\prime}\right)\right\rangle_{\text {stoc }}$ $=\gamma \hbar^{2} /(2 e)^{2} \delta\left(t-t^{\prime}\right)$, where $\langle\cdot\rangle_{\text {stoc }}$ defines the average over the fluctuations. Thus defined, $\gamma$ is just the inverse decoherence time of the two charge states. If we neglected the fluctuations, the time evolution of the system would be fully coherent, governed by the coherent part of the Hamiltonian $\hat{H}_{c}$ $\equiv\langle\hat{H}\rangle_{\text {stoc }}$. In this case the quantum state of the central grain would acquire dynamical phases $2 \theta=E_{J} t_{J} / \hbar$ during the Josephson contact times and $2 \chi=2 e V_{0} t_{C} / \hbar$ during the free evolution time (we assume that $V_{0}$ is present only during both intervals of the free evolution time).

With fluctuations, the shuttle will be described by a 2 $\times 2$ density matrix that obeys the following Bloch equation:

$$
\frac{\partial \hat{\rho}}{\partial t}=-\frac{i}{\hbar}\left[\hat{H}_{c}(t) \hat{\rho}-\hat{\rho} \hat{H}_{c}(t)\right]-2 \gamma\left(\hat{\rho}-\sigma_{z} \hat{\rho} \sigma_{z}\right) .
$$

The only stationary solution of this equation is quite trivial: $\hat{\rho} \propto \hat{1}$; this corresponds to the absence of an averaged superconducting current. Therefore, the shuttle operates in the incoherent regime. This is a combined effect of the decoherence term and Josephson coupling. In the absence of Josephson coupling, voltage fluctuations cannot cause transitions between the charge states so no relaxation takes place. With Josephson coupling, the voltage fluctuations cause transitions between the stationary states separated by energy $2 E_{J}$ at $V_{0}=0$. Classical voltage fluctuations result in equal transition rates with increasing and decreasing energy. In fact, the ratio of these rates is given by the Bolzmann factor $\exp \left(2 E_{J} / T_{b}\right), T_{b}$ being the temperature of the environment producing the fluctuations. As shown in Ref. 7, proper account of this factor leads to anisotropic $\hat{\rho} \neq \hat{1}$ and to the net supercurrent vanishing at $T_{b} \gg E_{J}$. This defines the incoherent regime under consideration.

Let us evaluate the FCS. One starts with the assumption that a transport process can be characterized by the probabilities $P_{\tau}(N)$ of $N$ electrons transferred through the contact in a time interval $\tau$ and attempts to compute the characteristic function of this probability distribution defined as ${ }^{2}$

$$
e^{-\mathfrak{G}(\lambda, \tau)}=\sum_{N} P_{\tau}(N) e^{i \lambda N}
$$

The general quantum expression for this function can be obtained by coupling the system to a detector for a measuring time $\tau$ and interpreting the detector readout in terms of charge transfer. ${ }^{5}$ The FCS in this case is defined as the integral kernel that relates the initial and final density matrices of the detector and reads

$$
e^{-\mathfrak{G}(\lambda, \tau)}=\operatorname{Tr} \underbrace{\left[\mathcal{U}_{+\lambda}(\tau, 0) \hat{\rho}(0) \mathcal{U}_{-\lambda}^{\dagger}(\tau, 0)\right]}_{\hat{\rho}^{\prime}(\tau)}
$$

where $\mathcal{U}_{\lambda}(\tau, 0)=\vec{T} \exp \left(-i \int_{0}^{\tau} \hat{H}_{\lambda}(s) d s\right)$ is the unitary evolution operator corresponding to the modified Hamiltonian $\hat{H}_{\lambda}=\hat{H}$ $+\lambda \hat{I} / e, \hat{I}$ being the operator of the electric current. Two such operators provide nonunitary evolution of the initial density matrix of the system $\hat{\rho}(0)$ into $\hat{\rho}^{\prime}(\tau)$.

For our shuttle model, the implementation of this general scheme is especially simple since the density matrix is just a $2 \times 2$ matrix. Following Ref. 4, we also gauge the counting field $\lambda$ to the left electrode; this yields $\hat{H}_{\lambda}=\hat{H}_{c}\left(\phi_{L} \rightarrow \phi_{L}+\lambda\right)$. We derive an equation for $\hat{\rho}^{\prime}$ that looks very similar to the Bloch equation (2): 


$$
\frac{\partial \hat{\rho}^{\prime}}{\partial t}=-\frac{i}{\hbar}\left(\hat{H}_{+\lambda} \hat{\rho}^{\prime}-\hat{\rho}^{\prime} \hat{H}_{-\lambda}\right)-2 \gamma\left(\hat{\rho}^{\prime}-\sigma_{z} \hat{\rho}^{\prime} \sigma_{z}\right)
$$

The difference is that Hamiltonians governing the evolution of bras and kets differ by opposite $\lambda$ shifts.

This system of four linear equations can be solved with initial conditions $\hat{\rho}^{\prime}\left(t_{0}\right)$ to obtain a linear map $M$ that gives $\hat{\rho}^{\prime}$ after a cycle, $\hat{\rho}^{\prime}\left(t_{0}+T\right)=M \hat{\rho}^{\prime}\left(t_{0}\right)$. All four eigenvalues $\mu_{i}$ of $M$ satisfy the condition $\left|\mu_{i}\right| \in[0,1]$. Since we study statistics of low-frequency fluctuations, $\tau \gg T$ by definition. Therefore, the FCS is determined by the eigenvalue with the greatest magnitude,

$$
e^{-\mathfrak{G}(\lambda, \tau)}=\bar{\mu}(\lambda)^{\tau / T} \quad \text { where } 1-\bar{\mu}=\min _{i=1, \ldots, 4}\left(1-\mu_{i}\right) .
$$

This is in accordance with the method and the result ${ }^{12}$ for the FCS of charge transport described by a master equation.

The most transparent way to present the FCS is to define the probabilities $p_{N}$ to transfer $N$ electrons per cycle, as was done in Ref. 9 to characterize the pumping of normal electrons,

$$
p_{N}=\frac{1}{2 \pi} \int_{-\pi}^{\pi} d \lambda e^{-i N \lambda} \bar{\mu}(\lambda)
$$

The FCS in the limit considered is always an even function of $\lambda$, so that the pairs are shuttled in either direction with equal probability, $p_{N}=p_{-N}$.

We analyze the FCS in several limiting cases. First we consider the limit of long periods $1 / t_{C}, 1 / t_{J} \ll \gamma \ll E_{J} / \hbar$. This is in fact an adiabatic limit since the relaxation and decoherence are fully developed within each time interval $t_{C}, t_{J}$. In this case,

$$
\bar{\mu}(\lambda)=\cos ^{2} \lambda \rightarrow p_{0}=\frac{1}{2}, \quad p_{ \pm 2}=\frac{1}{4},
$$

so that each shuttling between the superconductors transfers either one Cooper pair or none; this occurs with equal probabilities. The pair is transferred with equal probabilities in either direction. It is interesting to note that this simple result is quite general and relies on neither the periodicity of shuttling nor the concrete time dependence of $E_{J}(t)$ provided the adiabaticity is preserved. The shuttle may even return (several times) to the same superconducting terminal before contacting the opposite one: the charge transfer is in this case associated with two trips between the opposite terminals. Leading corrections to adiabatic FCS are exponentially small, $\simeq \exp \left(-2 t_{J} \gamma\right)$.

Another interesting limit is that of small Josephson couplings $E_{J} \ll \hbar \gamma$ and sufficiently long cycles $\hbar / t_{C}, \hbar / t_{J} \ll E_{J}$. In this case, the charge relaxation time $\simeq \gamma \hbar^{2} / E_{J}^{2}$ may be long, exceeding both decoherence time and cycle duration. The relevant parameter $\exp \left[-t_{J} E_{J}^{2} /\left(\hbar^{2} \gamma\right)\right] \equiv f$ determines the efficiency of the charge relaxation during a cycle. The FCS becomes more complicated; for $f \simeq 1$ it is given by
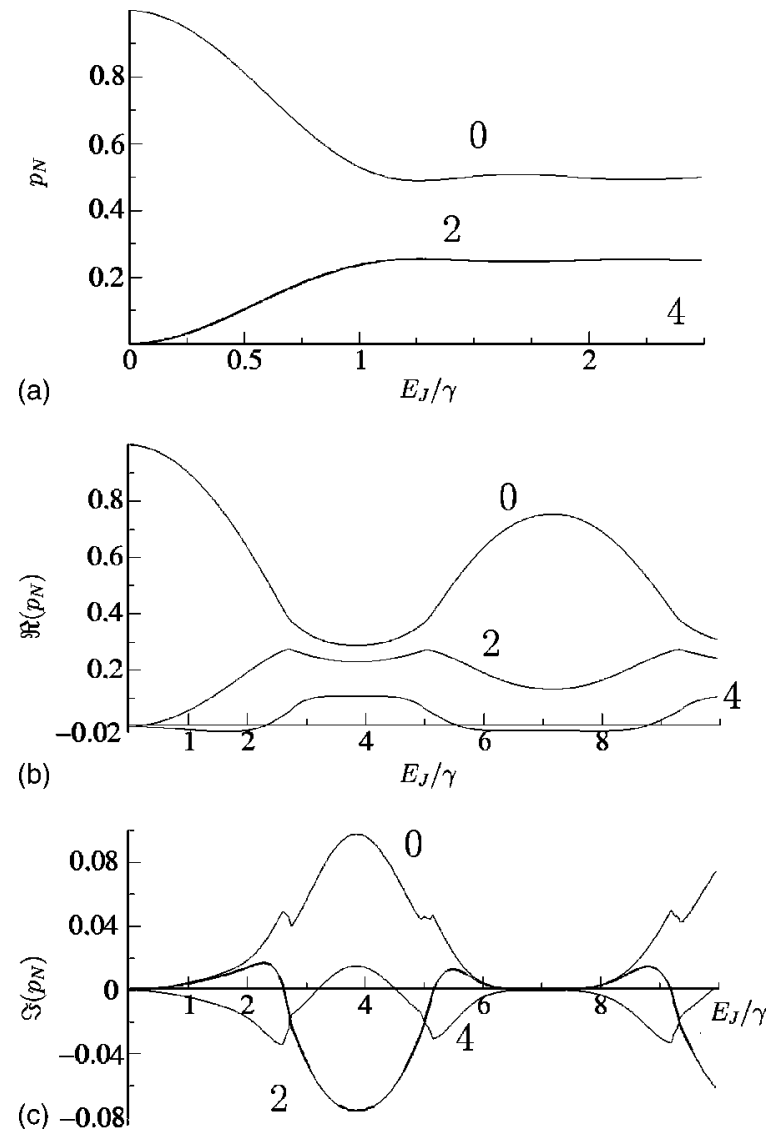

FIG. 2. Fourier components $p_{N}$ with $N=0,2,4$ of $\bar{\mu}(\lambda)$ plotted versus $E_{J} / \hbar \gamma$. Each line is labeled by the corresponding value of $N$. Adiabatic regime (a) $\gamma t_{J}=5$; positive probabilities. General situation (b)-(c) $\gamma t_{J}=0.9 ; p_{N}$ can be negative and even imaginary. Other parameters are fixed to $t_{J}=t_{C}, \chi=\pi / 5, \phi=\pi / 4$ for all plots.

$$
\begin{aligned}
\bar{\mu}(\lambda)= & \frac{1}{2}\left(1+f^{2}\right) \cos ^{2} \lambda+f \sin ^{2} \lambda+\left(1-f^{2}\right) \\
& \times \frac{1}{2} \sqrt{\cos ^{2} \lambda\left[1-\left(\frac{1-f}{1+f}\right)^{2} \sin ^{2} \lambda\right]},
\end{aligned}
$$

and, generally speaking, all $p_{N} \neq 0$. This is because the charge cannot completely relax during a cycle providing "memory effect" so that charge transfers in different cycles are not independent and an elementary event of charge transfer can encompass several cycles. Still, the probabilities remain positively defined owing to the fact that in the limit of long cycles the FCS depends neither on the superconducting phase $\phi$ nor on dynamical phases $\theta, \chi$.

Beyond this limit, the FCS does depend on $\phi$. As mentioned in Ref. 4, the classical interpretation in this case may fail and one cannot assure that $p_{n}$ are positive or even real. Indeed, we have found this in numerical calculations at $\gamma T$ $\simeq 1$ (Fig. 2). This clearly signals that the superconducting coherence remains in the system although the decoherence completely destroys the average supercurrent.

This becomes evident when analyzing the opposite limiting case of very short cycles, $\gamma T \ll 1$. In this case, the cumu- 


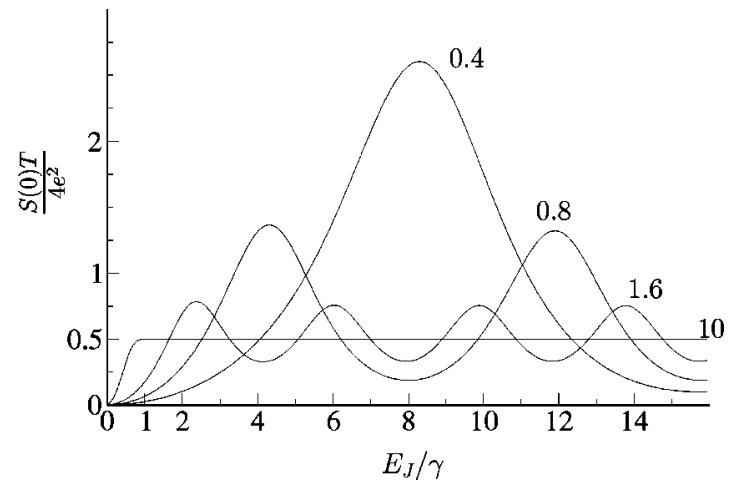

FIG. 3. Low-frequency noise in the intermediate regime for different values of $\gamma t_{J}$, as expressed by each line label. The noise and its oscillations with $\theta$ increase with decreasing $\gamma t_{J}$. Other parameters are fixed to $t_{J}=t_{C}, \chi=\pi / 5, \phi=\pi / 3$ for all plots.

lants of the current are contributed by FCS at $\lambda \simeq \gamma T \ll 1$. In this region, the FCS reads

$$
\frac{\bar{\mu}-1}{T} \simeq-\frac{\tilde{\gamma}}{2}+\sqrt{\left(\frac{\tilde{\gamma}}{2}\right)^{2}-\left(\frac{\lambda I_{s}}{e}\right)^{2}},
$$

where $I_{s}$ is the superconducting current in the absence of decoherence $\gamma=0$ and $\tilde{\gamma}$ is the relaxation time in the limit $\gamma \rightarrow 0$ :

$$
I_{s}=\frac{2 e}{T} \frac{\partial \alpha}{\partial \phi}, \quad \tilde{\gamma}=\frac{\gamma}{\sin ^{2} \alpha}\left(\frac{t_{J}}{T} P_{J}+\frac{t_{C}}{T} P_{C}\right),
$$

where $\alpha \equiv \arccos \left(\cos ^{2} \theta \cos 2 \chi-\sin ^{2} \theta \cos \phi\right)$ and $P_{J}, P_{C}$ are always positive polynomial functions in $\sin (\cdot)$ and $\cos (\cdot)$ of the phases $\phi, \chi, \theta$ :

$$
\begin{gathered}
P_{C}(\phi, \chi, \theta)=2 \sin ^{2}(2 \theta)[1+\cos \phi \cos (2 \chi)], \\
P_{J}(\phi, \chi, \theta)=2\left[1+\sin ^{2} \theta \cos \phi \cos (2 \chi)\right]-\cos (2 \theta) \\
\times\left[\cos ^{2} \theta \cos ^{2}(2 \chi)-\sin ^{2} \theta \cos ^{2} \phi\right] .
\end{gathered}
$$

FCS of this type corresponds ${ }^{4}$ to a supercurrent randomly switching at time scale $1 / \tilde{\gamma} \gg T$ between the opposite values $\pm I_{s}$, so that the coherence is preserved at this time scale. The charges are transferred in long trains of $\simeq 1 / \tilde{\gamma} T$ of elementary charge; this leads to significant low-frequency current noise.

Low-frequency noise $S(\omega=0)$ is obtained directly from the definition of the FCS, $S(0)=-\left(e^{2} / T\right) \lim _{\lambda \rightarrow 0} \partial^{2}\left(\bar{\mu} / \partial \lambda^{2}\right)$. From Eq. (9) we obtain $S(0)=\left(2 e^{2} / T\right) \times(1-f) /(1+f)$ in the adiabatic limit. The noise is enhanced in the opposite limit of short cycles, $S(0)=2 I_{s}^{2} / \tilde{\gamma} \gg e^{2} / T$, and is sensitive to all dynamical phases. The numerical results in the intermediate regime (Fig. 3) show in addition a quasioscillatory dependence on the dynamical phase $\theta$.

The authors appreciate many enlightening discussions with R. Fazio. This work was supported by the EU (ISTSQUBIT, Grant No. HPRN-CT-2002-00144) and by Fondazione Silvio Tronchetti Provera.
${ }^{1}$ B. D. Josephson, Phys. Lett. 1, 251 (1962).

${ }^{2}$ L. S. Levitov, H. W. Lee, and G. B. Lesovik, J. Math. Phys. 37, 4845 (1996).

${ }^{3}$ Quantum Noise in Mesoscopic Physics, edited by Yu. V. Nazarov (Kluwer, Dordrecht, 2002).

${ }^{4}$ W. Belzig and Yu. V. Nazarov, Phys. Rev. Lett. 87, 197006 (2001).

${ }^{5}$ M. Kindermann and Yu. V. Nazarov in Ref. 3, p. 403; see also M. Kindermann and Yu. V. Nazarov, Eur. Phys. J. B 35, 413 (2003).

${ }^{6}$ L. Y. Gorelik, A. Isacsson, Y. M. Galperin, R. I. Shekhter, and M.
Jonson, Nature (London) 411, 454 (2001).

${ }^{7}$ A. Romito, F. Plastina, and R. Fazio, Phys. Rev. B 68, 140502(R) (2003).

${ }^{8}$ R. I. Shekhter, Yu. Galperin, L. Y. Gorelik, A. Isacsson, and M. Jonson, J. Phys.: Condens. Matter 15, R441 (2003).

${ }^{9}$ L. S. Levitov, in Ref. 3.

${ }^{10}$ A. Andreev and A. Kamenev, Phys. Rev. Lett. 85, 1294 (2000).

${ }^{11}$ J. Tobiska and Yu. V. Nazarov, Phys. Rev. Lett. 93, 106801 (2004).

${ }^{12}$ D. A. Bagrets and Yu. V. Nazarov, Phys. Rev. B 67, 085316 (2003). 